\section{A practical guide to zoonotic diseases: what do I need to tell clients?}

\section{Jane E. Sykes}

Animal owners are exposed on a daily basis to microorganisms colonizing or infecting pets that can cause zoonotic disease. Veterinary surgeons are in a unique position to educate owners about risk factors for zoonoses and ways to prevent them.

Immunocompromised people are at greatest risk of acquiring zoonoses, and tend to develop more severe disease, a longer duration of illness and more severe complications. Up to $20 \%$ of the North American population and $60 \%$ of North American households has some degree of immunosuppression; similar prevalences are likely in Europe. In general, the positive health benefits of pet ownership are likely to outweigh the risks, so client education to reduce risks is key. In general, the incidence of pet-associated disease is low.

The most common routes of transmission are through bites, scratches, faecal-oral transmission, direct or indirect contact with body fluids, and vector-borne transmission. In general, young animals (puppies and kittens $<1$ year) are more likely to transmit zoonoses to people. The most common zoonoses are bite wound infections (Pasteurella, Capnocytophaga), dermatophytosis, bartonellosis, brucellosis, scabies, methicillinresistant Staphylococcus infections, enteropathogenic bacterial and protozoal infections (Campylobacter, Salmonella, Giardia, Cryptosporidium), and toxocariasis. Regional differences exist in regards to risk. For example, zoonotic transmission of Sporothrix has been of considerable concern in Brazil, leishmaniosis in South America and the Mediterranean, and rabies in developing countries in Africa and Asia. Some zoonotic infectious diseases are uncommon, but because they can cause severe illness or mortality in humans, in endemic regions, pet owner education may be important. Examples are plague, tularaemia, Rocky Mountain spotted fever, rabies and leptospirosis.

\section{ADVICE VETERINARY SURGEONS CAN PROVIDE TO OWNERS TO MINIMIZE RISK OF ZOONOTIC DISEASES}

- Choose healthy puppies and kittens. In particular puppies and kittens with diarrhoea or skin disease should be avoided by immunocompromised people. Immunocompromised people should ideally adopt dogs and cats that are at least 1 year of age and well socialized. Ownership of some exotic pets and especially reptiles (turtles, iguanas) should be avoided by the immunocompromised because of increased risk of salmonellosis

- Take measures to avoid bites, scratches and needle-stick injuries

- Ensure pets are vaccinated against rabies

- Wash hands properly after handling pets (especially important in immunocompromised individuals) and before eating. Instructions should be provided regarding proper handwashing techniques

- Use year-round ectoparasite and endoparasite preventatives

- Seek veterinary care promptly when pets become ill, and notify your healthcare provider if you develop illness in association with pet illness or a bite from a pet

- Inform your healthcare provider when your pet is diagnosed with a zoonotic disease

- Feed pets properly cooked food or commercial processed pet food diets; avoid raw meat diets

- Inspect dogs for ticks after hiking in tick-endemic regions. Remove ticks promptly with curved forceps or other tick-removal device. Avoid using bare hands to remove ticks.

\section{KEY LEARNING OBJECTIVES}

- Understand risk factors for zoonoses in pet owners

- Know the most common zoonotic infectious diseases of companion animals

- Know what to tell pet owners to minimize risk of them acquiring a zoonotic infectious disease

\section{MULTIPLE CHOICE QUESTIONS}

1. Approximately what proportion of households in North America are thought to be immunocompromised?
(A) $2 \%$
(B) $20 \%$
(C) $60 \%$
(D) $80 \%$

2. Which of the following zoonotic diseases is likely most common in pet owners?

(A) Leptospirosis

(B) Bite wound infections

(C) Cat-scratch disease

(D) Dermatophytosis

3. Which of the following exotic pets is least likely to be associated with transmission of salmonellosis to humans?
(A) Hedgehog
(B) Turtle
(C) Sugar glider
(D) Rabbit 